OPEN ACCESS

Edited by:

Ute Christiane Meier,

Ludwig Maximilian University of

Munich, Germany

Reviewed by:

Daniel Scott-Algara,

Institut Pasteur, France

Pedro Castro,

University of Porto, Portugal

*Correspondence:

Yujun Pan

yujunpan@ems.hrbmu.edu.cn

Specialty section:

This article was submitted to

Inflammation,

a section of the journal

Frontiers in Immunology

Received: 22 May 2021 Accepted: 05 January 2022 Published: 10 February 2022

Citation:

Qu Y, Yang F, Meng F, Chen X, Zhang Q, Yu T, Wen S and Pan Y

(2022) Plasma Concentration of Tumor Necrosis Factor-Stimulated Gene-6 as a Novel Diagnostic and 3Month Prognostic Indicator in NonCardioembolic Acute Ischemic Stroke.

Front. Immunol. 13:713379. doi: 10.3389/fimmu.2022.713379

\section{Plasma Concentration of} Tumor Necrosis Factor-Stimulated Gene- 6 as a Novel Diagnostic and 3-Month Prognostic Indicator in Non-Cardioembolic Acute Ischemic Stroke

\author{
Yewei Qu, Fan Yang, Fanwei Meng, Xi Chen, Qingqing Zhang, Tian Yu, Shirong Wen \\ and Yujun Pan *
}

Department of Neurology, the First Affiliated Hospital of Harbin Medical University, Harbin, China

Background: Tumor necrosis factor-stimulated gene-6 (TSG-6) is a multifunctional, antiinflammatory, and protective protein, while the association between TSG-6 and acute ischemic stroke (AIS) remains unclear in humans. This study aims to investigate the potential diagnostic and short-term prognosis predictive values of TSG-6 in noncardioembolic AIS.

Methods: A total of 134 non-cardioembolic AIS patients within $24 \mathrm{~h}$ after AIS onset and 40 control subjects were recruited. Using an AIS dataset from the Gene Expression Omnibus database and setting the median expression level of TNFAIP6 as the cutoff point, data were divided into TNFAIP6-high and TNFAIP6-low expression groups. Differently expressed genes (DEGs) were extracted to perform gene enrichment analysis and protein-protein interaction (PPI) network. Baseline data were analyzed in a four-group comparison plotted as plasma TSG-6 concentration median and 25th/75th percentiles. The correlative factors of 3-month outcome were evaluated by logistic regression. TSG-6 concentrations and TSG-6-to-interleukin-8 ratios were compared in a block design. A receiver-operating characteristic curve was used to analyze the detective value of TSG-6 and 3-month prognosis predictive values of TSG-6 and TSG-6-to-interleukin-8 ratio.

Results: Non-cardioembolic AIS patients had significantly higher plasma TSG-6 levels than control subjects $(P<0.0001)$. The large-artery atherosclerosis group had significantly higher TSG-6 levels than the small-artery occlusion group $(P=0.0184)$. Seven hundred and eighty-two DEGs might be both AIS-related and TNFAIP6-correlated genes, and 17 targets were deemed AIS-related being closely relevant to TNFAIP6. Interleukin-8 was selected for further study. The National Institutes of Health Stroke Scale and the Acute Stroke Registry and Analysis of Lausanne scores at admission, lesion volume, neutrophil count, neutrophil-to-lymphocyte ratio, and interleukin-8 level were positively correlated with TSG-6 level, respectively $(P<0.0001)$. The unfavorable outcome group had 
meaningfully higher TSG-6 levels $(P<0.0001)$ and lower TSG-6-to-interleukin-8 ratios $(P<0.0001)$ than the favorable outcome group. After adjusting for confounding variables, elevated TSG-6 levels remained independently associated with 3-month poor prognosis of non-cardioembolic AIS ( $P=0.017)$. In non-cardioembolic AIS, the cutoff values of TSG6 concentration for detection and 3-month prognosis prediction and the TSG-6-tointerleukin-8 ratio for the 3-month prognosis prediction were $8.13 \mathrm{ng} / \mathrm{ml}$ [AUC, 0.774 (0.686-0.861); $P<0.0001], 10.21 \mathrm{ng} / \mathrm{ml}$ [AUC, 0.795 (0.702-0.887); $P<0.0001$ ], and 1.505 [AUC, $0.873(0.795-0.951) ; P<0.0001]$.

Conclusions: Plasma TSG-6 concentration was a novel indicator for non-cardioembolic AIS diagnosis and 3-month prognosis. Elevated TSG-6-to-interleukin-8 ratio might suggest a 3-month favorable outcome.

Keywords: prognosis, protein-protein interaction network, neutrophil, acute ischemic stroke, interleukin-8, tumor necrosis factor-stimulated gene- 6

\section{BACKGROUND}

Stroke remains to be a major leading cause of global disease burden (1). From 1990 to 2019, the globally absolute number of incident strokes increased by $70 \%$, prevalent strokes increased by $85 \%$, deaths from stroke increased by $43 \%$, and disability-adjusted lifeyears due to stroke increased by $32 \%$ (1). In China, large amounts of medical and health resources have been devoted to patients with stroke (2). Meanwhile, around 795,000 people experience a firstever or recurrent stroke every year in the USA (3).

Acute ischemic stroke (AIS) triggers an inflammatory cascade accompanied with a series of functional modulations in inflammatory cells $(4,5)$. Tumor necrosis factor-stimulated gene-6 (TSG-6) encoded by the TNFAIP6 gene is a multifunctional secretory protein exhibiting anti-inflammatory and tissue protective properties and being implicated in certain physiological processes and some disease pathology (6). Clinical studies have indicated that plasma TSG-6 levels evidently increased in patients with acute coronary syndrome (ACS) (7) and abdominal aortic aneurysms (AAA) (8). In addition, animal studies, as well as humans studies, showed increasing expression of TSG-6 in infarcted brain tissues $(9,10)$.

Given these findings above, plasma TSG-6 levels may be associated with the occurrence and prognosis of AIS. However, to date, there is little information on the association between

\footnotetext{
Abbreviations: TSG-6/TNFAIP6, tumor necrosis factor-stimulated gene-6; AIS, acute ischemic stroke; IL-8: interleukin-8; SD, standard deviation; OR, odds ratio; $\mathrm{CI}$, confidence interval; IQR, interquartile range; ROC, receiver-operator characteristic; AUC, area under the curve; LLA, large-artery atherosclerosis; SAO, small-artery occlusion; ELISA, enzyme-linked immunosorbent assay; mRS, modified Rankin Scale; BMI, body mass index; NIHSS, National Institutes of Health Stroke Scale; ASTRAL, Acute Stroke Registry and Analysis of Lausanne; SBP, systolic blood pressure: DBP, diastolic blood pressure; NLR, neutrophil-tolymphocyte ratio; FBG, fasting blood glucose; LDL, low-density lipoprotein; HDL, high-density lipoprotein; APOA, apolipoprotein A; APOB, apolipoprotein B; Lpa, Lipoprotein(a); HCY, homocysteine; UA, uric acid; DAPT, dual antiplatelet therapy; mTICI, modified thrombolysis in cerebral infarction; rt-PA, recombinant tissue plasminogen activator; u-PA, urokinase plasminogen activator; KEGG, Kyoto Encyclopedia of Genes and Genomes; GO, Gene Ontology; PPI, protein-protein interaction; DEGs, differentially expressed genes.
}

circulating TSG-6 and AIS in human. To better understand an emerging role of TSG-6 in non-cardioembolic AIS, we prospectively investigated their association, including the values on the diagnosis and prognosis.

\section{METHODS}

The flowchart in this study is shown in Figure 1.

\section{Study Population and Evaluation of Clinical Data}

A cross-sectional study of the association between plasma TSG-6 levels and non-cardioembolic AIS was conducted. A longitudinal descriptive study was conducted among the non-cardioembolic AIS. The recruitment period was 6 months, from July to December in 2020, in the neurology department at the First Affiliated Hospital of Harbin Medical University. Our study was approved by the local Ethics Committee of the First Affiliated Hospital of Harbin Medical University. AIS was diagnosed according to the World Health Organization criteria (11). AIS patients within 24 hours from symptoms onset were enrolled in the analysis. The exclusion criteria included symptom over $24 \mathrm{~h}$, new cerebrovascular events or ACS within 6 months, sequelae of stroke, major trauma or surgery within 3 months, severe organ failure, vascular malformation, malignant tumors, systemic inflammatory and autoimmune diseases, active infection/take antibiotics or antivirals within a week, cardioembolic/undetermined/other strokes, inadequate/absent MRI and lost to follow-up at 90 days. During the same period, people who aimed to take the physical examination in our ward, were collected as healthy control subjects. The exclusion criteria of healthy control group were the same as that of non-cardioembolic AIS group. Stata v15.1 (Stata Corp, College Station, TX) (12) was used to match sex and age between control subjects and non-cardioembolic AIS patients. All participants or their relatives in our study were informed of the study and signed the informed consents.

The clinical characteristics of the patients included sex, age, body mass index (BMI), history of risk factors [including a previous 


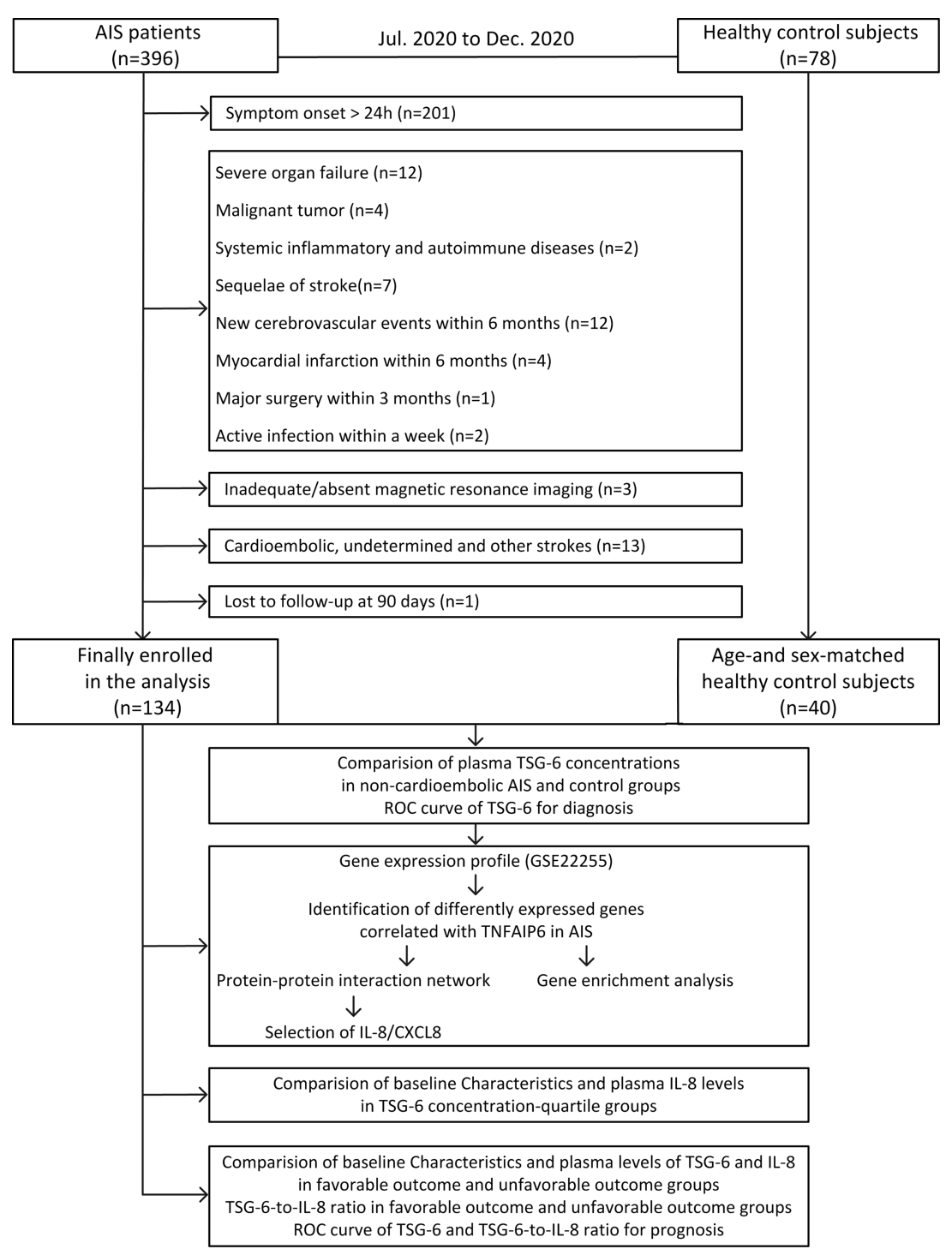

FIGURE 1 | Study flowchart. AIS, acute ischemic stroke; TSG-6, tumor necrosis factor-stimulated gene-6; Roc,receiver-operating characteristic curve; IL-8, interleukin-8.

transient ischemic attack (TIA) or symptomatic stroke, hypertension, diabetes mellitus, cardiovascular disease (CVD), alcohol drinking, current smoker], and blood pressure [including systolic blood pressure (SBP), diastolic blood pressure (DBP)]. Neurological deficit at admission was assessed using the National Institutes of Health Stroke Scale (NIHSS) (13) and the Acute Stroke Registry and Analysis of Lausanne (ASTRAL) score (14) to reflect the severity of AIS. According to the Trial of Org 10172 in the Acute Stroke Treatment (TOAST) system (15), non-cardioembolic AIS is divided into large-artery atherosclerosis (LAA) or small-vessel occlusion (SVO). The lesion volume was calculated using the formula $0.5 \times a \times b \times c$ (16). In detail, " $a$ " represents the maximal longitudinal largest diameter, " $b$ " represents a second maximal diameter perpendicular to $a$, and " $c$ " represents the height of the ellipsoid (5-mm slices containing the infarct were used in the present study). All blood examinations were determined at the Department of Laboratory Medicine of our hospital blinded to baseline data. Acute therapies included mono-antiplatelet therapy, dual antiplatelet therapy (DAPT), statin therapy, intravenous thrombolysis, and endovascular therapy (mechanical thrombectomy, angioplasty, and stenting). Modified thrombolysis in cerebral infarction (mTICI) score was used to assess recanalization after ET and successful reperfusion signified filling of $50 \%$ or more of the downstream territory ( $\geq$ mTICI $2 b)(17)$.

\section{Determination of Circulating TSG- 6 and Interleukin-8 (IL-8/CXCL8) Concentration}

The cubital venous blood of $3 \mathrm{ml}$ in the AIS group was collected on admission within $24 \mathrm{~h}$ from stroke onset, and fasting venous blood was collected into EDTA tubes in the control group. All 
blood samples were collected into EDTA tubes. After standing for $30 \mathrm{~min}$ and centrifugation at $1,500 \mathrm{rpm}$ for $20 \mathrm{~min}$ at $4^{\circ} \mathrm{C}$, the plasma of the samples was stored at $-80^{\circ} \mathrm{C}$ before the assay immediately. Double antibody sandwich enzyme-linked immunosorbent assay (ELISA) was performed following the instructions of human TSG-6 ELISA kit (RayBiotech, USA) and human IL- 8 ELISA kit (ABclonal, China). The results were measured with EMax Plus microplate reader by Molecular Devices. Standard curves (Supplementary Figure 1) were drawn for calculating the concentrations of TSG-6 and IL-8. Experienced laboratory technicians who measured TSG-6 and IL-8 concentrations were blinded to the clinical characteristics and outcomes of the participants.

\section{Data Processing and Identification of Differentially Expressed Genes}

The normalized GSE22255 expression data were downloaded from the Gene Expression Omnibus (GEO) database (http:// www.ncbi.nlm.nih.gov/geo/) (18). The series of GSE22255 is based on the GPL570 platform (Affymetrix Human Genome U133 Plus 2.0 Array). We extracted the expression data of 20 AIS samples from the GSE22255 dataset. After annotating gene symbols of probes on the GPL570 platform and filtering out the probes without corresponding gene symbols or with multiple genes, we averaged the expression value of genes that have multiple probes mapped. Differential expression analysis was performed using a limma package in $\mathrm{R}$ (19). The AIS patients were divided into TNFAIP6-high and TNFAIP6-low expression groups after setting the median expression level of TNFAIP6 as the cutoff point. The differentially expressed genes (DEGs) between TNFAIP6-high and TNFAIP6-low were identified using lmFit and eBayes functions in limma package. The cutoff value was set at $P<0.05$ adjusted by the false discovery rate (FDR) and the absolute value of $\log _{2}$-fold change ( $\mid \log _{2}$ $\mathrm{FC} \mid) \geq 0.5$.

\section{Gene Enrichment Analysis and Protein- Protein Interaction Network Construction}

The Metascape online tool (http://metascape.org/gp/ index.html\#/main/step1) (20) was applied to perform the Kyoto Encyclopedia of Genes and Genomes (KEGG) pathway and Gene Ontology (GO) function (biological process, cellular component, and molecular function) analysis for the enrichment analysis of the DEGs. The cutoff value was set at $P<0.05$. The corresponding protein-protein interaction (PPI) network of the DEGs was constructed by STRING (https://string-db.org/) (21). Subsequently, the network was visualized and further processed to assess the core targets interacted with TSG-6/TNFAIP6 by Cytoscape software (v3.6.1, http://www.cytoscape.org/) (22). A node with a high degree of closeness values indicates that the node plays a crucial role in the network. IL-8 was selected for further study.

\section{Follow-Up and End Point}

A 3-month follow-up was performed using the modified Rankin Scale (mRS) (23) by a trained medical staff blinded to clinical and neuroimaging data. At the time of discharge, $\mathrm{mRS}$ score from 0 to 2 was defined as a favorable outcome, whereas more than 2 points was adverse.

\section{Statistical Analysis}

The results are expressed as percentages for categorical variables, the means \pm standard deviation (SD) for normal continuous variables, and medians [interquartile ranges (IQRs)] for nonnormal continuous variables. Continuous data were checked for normal distribution using the Kolmogorov-Smirnov test and Shapiro-Wilk test. Homogeneity of variance used Levene's test. Proportions were compared using the chi-squared test. Normally distributed continuous variables with equal variance in the two datasets were analyzed by Student's $t$-test; if not, the Mann-Whitney $U$ test was used. A four-group comparison was plotted as plasma TSG-6 concentration median and 25th/ 75th percentiles. The normally distributed continuous variables with equal variance used the Student-Newman-Keuls $q$ test, and the Kruskal-Wallis test was used for statistical evaluation of the rest variables. For bivariate correlations, Spearman's rank correlation was used. Comparison between the different outcomes was investigated with logistic regression models. We used crude models and multivariable models adjusted for all predictors significant on univariate analysis, and the results were reported as odds ratios (ORs). The receiver-operating characteristic (ROC) curve was used to analyze the predictive value of plasma TSG-6 concentration and 3-month prognosis predictive values of plasma TSG- 6 concentration and TSG-6-to-interleukin-8 ratio in non-cardioembolic AIS, and the results were reported as area under the curve (AUC). Two-tailed $P<0.05$ was considered statistically significant. All statistical analyses and graphs were conducted by Statistical Package for Social Sciences (SPSS) version 22 (IBM Corp., Armonk, NY, USA) and GraphPad Prism software version 6.

\section{RESULTS}

\section{Baseline Characteristics and Plasma TSG- 6 Levels in Non-Cardioembolic AIS}

In this period, a total of 396 AIS patients were confirmed. Finally, after screening a consecutive range of AIS patients by inclusion and exclusion criteria and follow-up completion, 262 patients were excluded (Figure 1) and 134 non-cardioembolic AIS patients were enrolled. During the same period, 78 were collected as healthy control subjects, and of these patients, 40 age- and sex-matched people were included in our study.

The baseline characteristics of non-cardioembolic AIS patients are presented in Supplementary Table 1. At admission, the median TSG-6 concentration in noncardioembolic AIS group was 9.65 (IQR, 8.71-10.73) ng/ml and significantly higher than that of the controls [7.90 (IQR, 6.26-9.31) ng/ml; $P<0.0001$; Figure 2A], suggesting that high TSG-6 level was associated with non-cardioembolic AIS. The cutoff value for detecting non-cardioembolic AIS $(8.13 \mathrm{ng} / \mathrm{ml})$ by the ROC curve gained a relatively high true-positive rate 

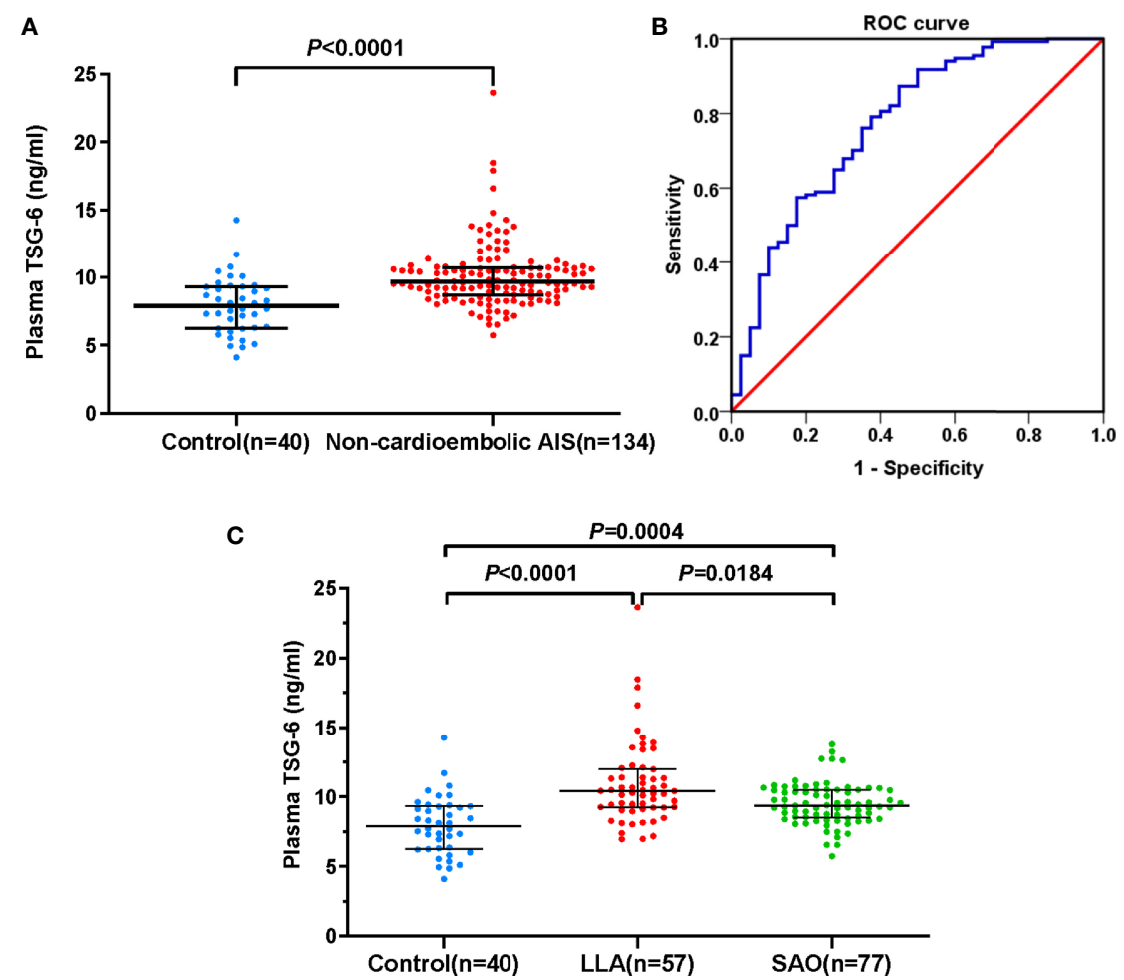

FIGURE 2 | Plasma TSG-6 levels. (A) Control subjects and non-cardioembolic AIS. (B) The ROC curve of plasma TSG-6 concentration for detecting non-cardioembolic AIS. The AUC value is $0.774(95 \% \mathrm{Cl}, 0.686-0.861 ; P<0.0001)$. (C) Control subjects, LLA, and SAO AIS. Horizontal lines represent median levels and interquartile ranges. $P$-values determined by the Mann-Whitney $U$ test (A) and Kruskal-Wallis test (C). AIS, acute ischemic stroke; TSG-6, tumor necrosis factor-stimulated gene-6; LLA, large-artery atherosclerosis; SAO, small-artery occlusion; ROC, receiver-operating characteristic; AUC, area under the curve.

(sensitivity, 0.873) with a moderate false-positive rate (1specificity, 0.450). The AUC value was 0.774 (95\% CI, 0.686$0.861 ; P<0.0001$; Figure 2B). According to the TOAST classification, 57 and 77 exhibited LAA and SAO. In the LLA group, there were $36(63.16 \%)$ with only intracranial stenosis, 3 (5.26\%) with only extracranial stenosis, and 18 (31.58\%) with both intracranial and extracranial stenosis. Comparing the LLA [10.78 (IQR, 10.42-12.25) ng/ml; $P<0.0001$; Figure 2C) or SAO [9.35 (IQR, 8.49-10.50) ng/ml; $P=0.0004$; Figure 2C] groups with the control group, the plasma TSG-6 levels of both subtypes were significantly higher. Additionally, the TSG-6 levels in LLA patients significantly increased than those in SAO patients $(P=0.0184$; Figure 2C).

\section{Identification of DEGs Correlated With TNFAIP6 in AIS, Gene Enrichment Analysis, and Integration of the PPI Network}

The normalized GSE22255 expression matrix containing 54,675 probes was determined after annotation based on the GPL570 platform. After setting the cutoff value as $P<0.05$ adjusted by the FDR and $\left|\log _{2} \mathrm{FC}\right| \geq 0.5$, a total of 782 DEGs, consisting of 466 upregulated and 316 downregulated genes, were screened out, which may be AIS-related genes that correlate with TNFAIP6 expression (Figure 3A and Supplementary Data Sheet 1).

Subsequently, KEGG analysis indicated that the DEGs were involved in pathways associated with response to immunoinflammatory response (NOD-like receptor signaling pathway, IL-17 signaling pathway, c-type lectin receptor signaling pathway, Toll-like receptor signaling pathway, RIG-Ilike receptor signaling pathway, chemokine signaling pathway), environmental information processing (TNF signaling pathway, NF-kappa B signaling pathway, cytokine-cytokine receptor interaction), and fluid shear stress and atherosclerosis (Figure 3B). Besides, the related biological processes consist of response to biotic stimulus and lipid, leukocyte functional activity, and regulation of cell death and cytokine production. The molecular functions were found mainly in transcription, kinase binding, protein heterodimerization activity, and activity or receptor binding of cytokine and chemokine (Figure 3C).

After constructing the PPI network of the DEGs with a threshold of medium confidence not less than 0.4 by STRING and then processing by the Cytoscape software, 17 targets at firststage nodes were deemed as closely interacting with TSG-6/ TNFAIP6 in AIS. We used the cytoHubba plugin to calculate the degree of these targets and the top 3 were CXCL1, CXCL8/IL-8, and EGF (Figure 3D and Supplementary Table 2). 
A

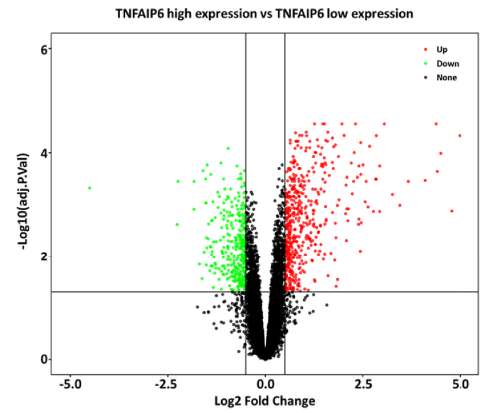

C

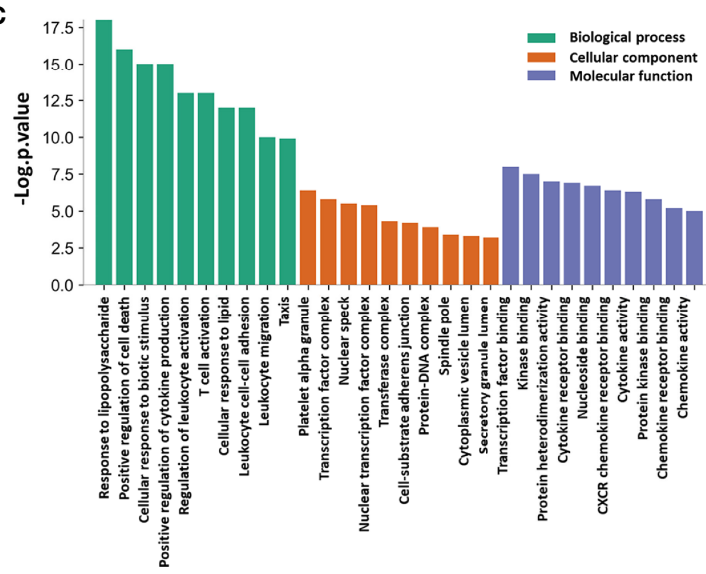

B

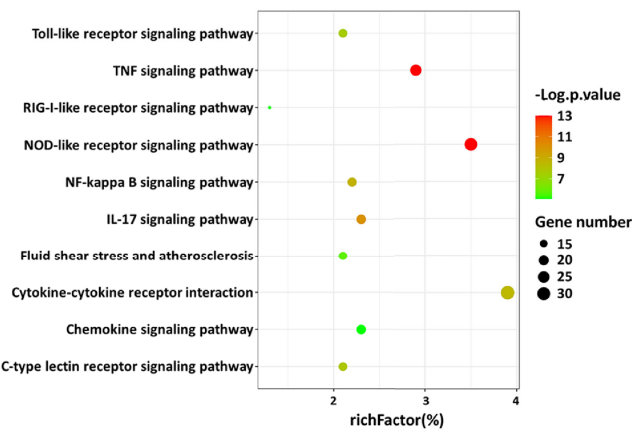

D

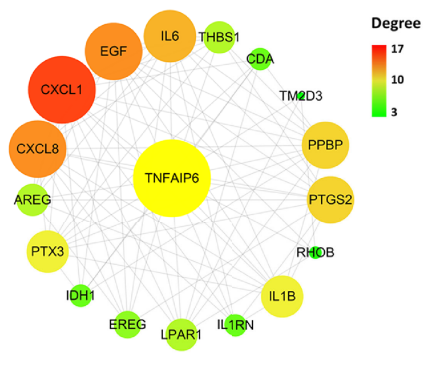

FIGURE 3 | Analysis of differently expressed genes. (A) Volcano plot of TNFAIP6 low/high: green represents downregulated genes, red represents upregulated genes, and black represents no significant differently expressed genes. (B) KEGG pathways analysis: the enriched significance gradually increases from green to red, and the dot sizes indicate the number of differential genes contained in the corresponding pathway. (C) Enriched GO terms. (D) Protein-protein interaction network: circles represent gene-encoded proteins, the related degree gradually increases from green to red, and the circle sizes indicate the target-related degree to TNFAIP. AIS, acute ischemic stroke; KEGG, Kyoto Encyclopedia of Genes and Genomes; GO, Gene Ontology; PPI, protein-protein interaction.

\section{Baseline Characteristics and Clinical Variables in Relation to TSG-6 Level}

Baseline characteristics of non-cardioembolic AIS patients were stratified in comparison to TSG-6 quartiles in Supplementary Table 1. The median concentration of TSG-6 was 9.65 (IQR, 8.71-10.73) ng/ml. Twenty-five percent of the patients had a TSG-6 concentration $>10.73 \mathrm{ng} / \mathrm{ml}$, defining the upper quartile. Compared with patients with lower TSG-6 levels, those with higher TSG-6 levels had significant higher NIHSS and ASTRAL scores at admission, lesion volumes, neutrophil counts, neutrophil-to-lymphocyte ratios (NLRs), levels of HCY and IL-8, and lower lymphocyte counts. Plasma TSG-6 level was moderately positively correlated with NIHSS score at admission $(r=0.493$, $P<0.0001)$ and ASTRAL score at admission $(r=0.434, P<0.0001)$, lesion volume $(r=0.359, P<0.0001)$, neutrophil count $(r=0.357$, $P<0.0001)$, and NLR $(r=0.368, P<0.0001)$. Moreover, there was a more significant positive correlation between the levels of TSG-6 level and those of IL-8 level $(r=0.639, P<0.0001)$. There was a weak negative correlation between the levels of TSG-6 and those of lymphocyte count $(r=-0.226, P=0.009)$. Statistical analysis revealed no significant correlations between TSG-6 level and those of HDL-c and HCY levels (Table 1).
TABLE 1 | Analysis of relative factors of TSG-6 plasma level in non-cardioembolic AIS patients.

\begin{tabular}{lcc}
\hline Relevant Factors & $\boldsymbol{r}_{\mathbf{s}}$ & $\boldsymbol{P}$-value \\
\hline NIHSS at admission & 0.493 & $<0.0001^{*}$ \\
ASTRAL at admission & 0.434 & $<0.0001^{*}$ \\
Lesion volumes & 0.359 & $<0.0001^{*}$ \\
Neutrophil & 0.357 & $<0.0001^{*}$ \\
Lymphocyte & -0.226 & $0.009^{*}$ \\
Neutrophil-to-lymphocyte ratio & 0.368 & $<0.0001^{*}$ \\
HDL-c & -0.002 & 0.984 \\
Homocysteine & 0.134 & 0.123 \\
Interleukin-8 & 0.639 & $<0.0001^{*}$
\end{tabular}

${ }^{*} P<0.05$.

AIS, acute ischemic stroke; TSG-6, tumor necrosis factor-stimulated gene-6; NIHSS, National Institutes of Health Stroke Scale; ASTRAL, Acute Stroke Registry and Analysis of Lausanne; HDL-c, high-density lipoprotein-cholesterol.

\section{Associations Between Plasma TSG-6 Level and Non-Cardioembolic AIS Prognosis}

An unfavorable outcome was found in $35(26.12 \%)$ patients with an mRS score over 2, and 99 patients got favorable outcomes. In univariate logistic regression analysis, we calculated the ORs of TSG-6 (OR $=1.157 ; 95 \%$ CI, 1.249-1.975; $P<0.0001)$ and other 
risk factors as presented in Table 2. After adjusting for confounding variables (age, alcohol drinking, NIHSS and ASTRAL score at admission, lesion volume, neutrophil and lymphocyte counts, NLR, D-dimer, triglycerides, apolipoprotein A, IL-8, DAPT, and ET), TSG-6 concentration remained an independent predictor of 3month poor prognosis $(\mathrm{OR}=2.171 ; 95 \% \mathrm{CI}, 1.152-4.093$; $P=0.017)$ by multivariable logistic analysis. In addition, lesion volume, neutrophil and lymphocyte counts, NLR, and IL-8 level remained independently significant outcome predictors (Table 2). In patients with unfavorable functional outcomes, plasma TSG-6 levels were meaningfully higher compared with those in patients with favorable outcomes [10.78 (IQR, 10.42-12.25) vs. 9.28 (IQR, 8.44-10.34) ng/ml; $P<0.0001$; Figure 4A).

With an AUC of 0.795 (95\% CI, 0.702-0.887; $P<0.0001$ ), TSG-6 concentration showed a significant discriminatory ability to assess non-cardioembolic AIS prognosis (Figure 4B), and $10.21 \mathrm{ng} / \mathrm{ml}$ (sensitivity, $0.829 ; 1$-specificity, 0.283 ) was the cutoff value with a higher true-positive rate and a lower false-positive rate for predicting 3-month outcome in non-cardioembolic AIS. Interestingly, TSG-6-to-IL-8 ratios were significantly higher in the favorable outcome group compared with those in the unfavorable functional outcome group [1.94 (IQR, 1.68-2.39] vs. 1.22 (IQR, $0.38-1.54) \mathrm{ng} / \mathrm{ml} ; P<0.0001$; Figure $4 \mathrm{C}$ ). The cutoff value of TSG-6-to-IL-8 ratio for predicting the 3-month prognosis of non-cardioembolic AIS (1.505) by ROC curve gained a relatively high true-positive rate (sensitivity, 0.743) and a low false-positive rate (1-specificity, 0.081). The AUC value was 0.873 (95\% CI, 0.795-0.951; $P<0.0001$; Figure 4D).

\section{DISCUSSION}

This is the first study to investigate the association between circulating TSG- 6 and stroke in humans. In the end, we selected

TABLE 2 | Logistic regression analysis for the 3-month outcome.

\begin{tabular}{|c|c|c|c|c|}
\hline \multirow[t]{2}{*}{ Parameter } & \multicolumn{2}{|c|}{ Univariate Analysis } & \multicolumn{2}{|c|}{ Multivariable Analysis } \\
\hline & OR $(95 \% \mathrm{Cl})$ & $P$-value & OR $(95 \% \mathrm{Cl})$ & $P$-value \\
\hline Sex & $1.544(0.672-3.550)$ & 0.304 & & \\
\hline Age & $1.051(1.008-1.095)$ & 0.019 & $1.150(0.956-1.382)$ & 0.138 \\
\hline $\mathrm{BMl}$ & 0.987 (0.879-1.108) & 0.825 & & \\
\hline Previous stroke/TIA & $1.250(0.571-2.736)$ & 0.577 & & \\
\hline Hypertension & $1.095(0.488-2.460)$ & 0.826 & & \\
\hline Diabetes mellitus & $1.576(0.697-3.563)$ & 0.275 & & \\
\hline Cardiovascular disease & $1.248(0.490-3.175)$ & 0.643 & & \\
\hline Alcohol drinking & $0.418(0.189-0.924)$ & 0.031 & $0.975(0.048-19.774)$ & 0.987 \\
\hline Current smoker & $0.912(0.420-1.979)$ & 0.815 & & \\
\hline SBP & $0.996(0.981-1.012)$ & 0.635 & & \\
\hline DBP & $0.976(0.950-1.004)$ & 0.092 & & \\
\hline NIHSS at admission & $1.275(1.167-1.393)$ & $<0.0001^{\star}$ & $1.011(0.416-2.457)$ & 0.980 \\
\hline ASTRAL at admission & $1.229(1.143-1.322)$ & $<0.0001^{\star}$ & $1.12(0.488-2.606)$ & 0.779 \\
\hline Lesion volume & $1.017(1.009-1.025)$ & $<0.0001^{\star}$ & 1.042 (1.013-1.073) & $0.005^{\star}$ \\
\hline Neutrophil & $1.485(1.239-1.781)$ & $<0.0001^{\star}$ & $8.644(1.482-50.422)$ & $0.017^{\star}$ \\
\hline Lymphocyte, $10^{9} / \mathrm{L}$ & $0.160(0.061-0.420)$ & $<0.0001^{\star}$ & $0.000(0.000-0.043)$ & $0.013^{\star}$ \\
\hline Neutrophil-to-lymphocyte ratio & $1.253(1.106-1.419)$ & $<0.0001^{\star}$ & 0.155 (0.030-0.798) & $0.026^{\star}$ \\
\hline Platelet & $0.993(0.986-1.000)$ & 0.061 & & \\
\hline Fibrinogen & $1.320(0.810-2.152)$ & 0.265 & & \\
\hline D-dimer & $3.113(1.681-5.762)$ & $<0.0001^{\star}$ & $1.458(0.643-3.310)$ & 0.367 \\
\hline FBG & $1.054(0.919-1.208)$ & 0.452 & & \\
\hline Triglycerides & $0.549(0.321-0.939)$ & $0.029^{*}$ & $1.156(0.139-9.598)$ & 0.893 \\
\hline $\mathrm{TC}$ & $0.852(0.607-1.194)$ & 0.351 & & \\
\hline LDL-C & $0.918(0.584-1.444)$ & 0.712 & & \\
\hline HDL-C & $0.443(0.107-1.841)$ & 0.263 & & \\
\hline LDL-c-to-HDL-c ratio & $1.056(0.651-1.714)$ & 0.826 & & \\
\hline APOA & $0.142(0.025-0.806)$ & $0.028^{*}$ & $0.037(0.000-25.781)$ & 0.323 \\
\hline APOB & $0.880(0.204-3.787)$ & 0.863 & & \\
\hline APOA-to-APOB ratio & $0.343(0.101-1.166)$ & 0.087 & & \\
\hline Lipoprotein(a) & 1.009 (0.995-1.022) & 0.206 & & \\
\hline Homocysteine & $0.993(0.962-1.026)$ & 0.675 & & \\
\hline Uric acid & $1.000(0.996-1.004)$ & 0.954 & & \\
\hline Interleukin-8 & $1.691(1.331-2.149)$ & $<0.0001^{\star}$ & $1.638(1.064-2.522)$ & $0.025^{\star}$ \\
\hline TSG-6 & $1.157(1.249-1.975)$ & $<0.0001^{\star}$ & $2.171(1.152-4.093)$ & $0.017^{\star}$ \\
\hline Dual antiplatelet therapy & $0.162(0.036-0.720)$ & $0.017^{\star}$ & $0.264(0.002-42.701)$ & 0.608 \\
\hline Intravenous thrombolysis & $2.290(0.880-5.959)$ & 0.090 & & \\
\hline Endovascular therapy & $6.024(2.111-17.192)$ & $0.001^{*}$ & $0.404(0.017-9.835)$ & 0.578 \\
\hline
\end{tabular}

${ }^{*} P<0.05$.

OR, odds ratio; Cl, confidence interval; BMI, body mass index; SBP, systolic blood pressure; DBP, diastolic blood pressure; NIHSS, National Institute of Health Stroke Scale; ASTRAL, Acute Stroke Registry and Analysis of Lausanne; FBG, fasting blood glucose; TC total-cholesterol; LDL, low-density lipoprotein-cholesterol; HDL, high-density lipoprotein-cholesterol; APOA, apolipoprotein A; APOB, apolipoprotein B; TSG-6, tumor necrosis factor-stimulated gene-6. 
A
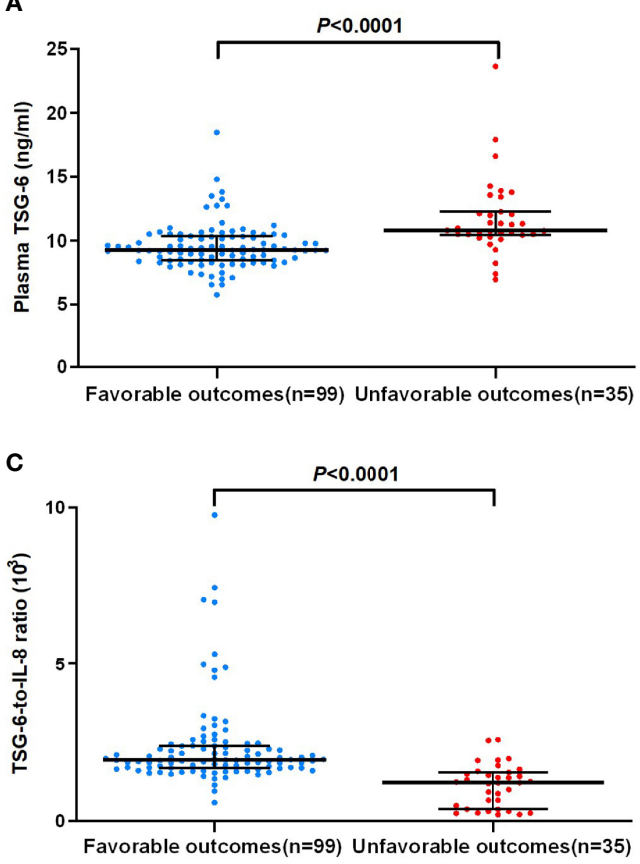

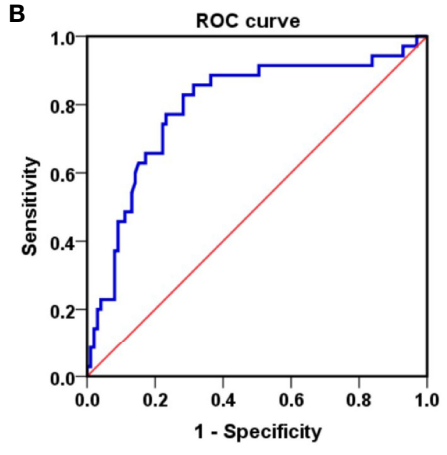

D

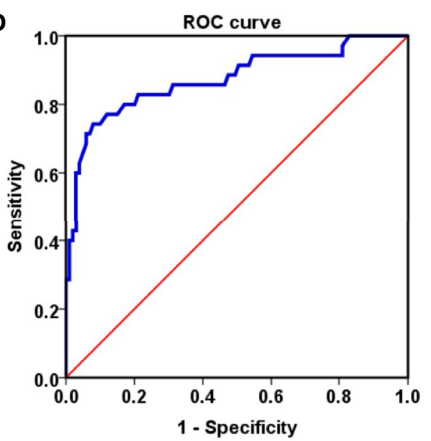

FIGURE 4 | (A) Plasma TSG-6 levels between favorable outcome and unfavorable outcome groups in non-cardioembolic AIS. (B) The ROC curve of plasma TSG-6 concentration for predicting 3-month prognosis of non-cardioembolic AIS. The AUC value is 0.795 (95\% Cl, 0.702-0.887; P<0.0001). (C) TSG-6-to-IL8 ratios between favorable outcome and unfavorable outcome groups in non-cardioembolic AIS. (D) The ROC curve of TSG-6-to-IL-8 ratio for predicting 3 -month-prognosis of non-cardioembolic AIS. The AUC value is $0.873(95 \% \mathrm{Cl}, 0.795-0.951 ; P<0.0001)$. The horizontal lines indicate median levels and interquartile ranges. $P$-values determined by the Mann-Whitney $U$ test. AIS, acute ischemic stroke; TSG-6, tumor necrosis factor-stimulated gene-6; IL-8, interleukin-8; ROC, receiver-operator characteristic.

non-cardioembolic stroke patients as the study population, mainly because there were too few patients with cardioembolic stroke that could be included in the study. Given this, cardioembolic, undetermined, and other stroke subtypes were removed. This proportion of subtype was in line with that in Asian populations. The proportion of cardioembolic stroke was about $10 \%$ in Chinese patients (2). We found that plasma TSG-6 levels in non-cardioembolic AIS patients significantly increased than those in controls. Likewise, Watanabe et al. (7) showed that plasma TSG-6 levels were significantly elevated in ACS. Moreover, the presence of TSG-6 expression was observed in the abdominal tunica media of AAA patients (8). TSG-6 is generally upregulated wherever there is inflammation to mediate many of immunomodulatory and reparative activities (6). We speculate that TSG-6 may act as a downregulator of the negative effects carried by proinflammatory cytokines to suppress an overreactive inflammation and maintain the dynamic balance of inflammatory mediator release in AIS. Further multiple comparisons between the control, LLA, and SVO groups showed significant increases of TSG-6 levels in both stroke subtypes, and the LAA group had meaningfully higher levels than the SAO group. Inflammation is well-known throughout the process of atherosclerotic plaque formation, destabilization, and rupture (24). TSG-6 contributes to prevent atherosclerosis by suppressing the inflammatory responses and oxidized LDL-induced foam cell formation and the migration and proliferation of vascular smooth muscle cells (25). Additionally, both animal and human studies have shown the expression of TSG-6 in atherosclerotic lesions, for instance, its localization in rat neointima after arterial injury, rabbit carotid atherosclerotic plaques, and human atherosclerotic fibrous cap $(7,8,26,27)$. We speculate that elevated circulating TSG-6 may reflect the activation of ongoing inflammation processes due to ruptured atherosclerotic plaque. As shown in Figure 1, the level differences between LLA patients and control subjects were greater than the differences between SVO patients and control subjects. Therefore, we hold the opinion that, for one thing, the overall severity of the condition of LLA patients was higher than that of SVO patients; for another, the atherosclerosis degree of LLA stroke was probably more serious than SVO stroke.

Our present study had suggested that a high expression of TSG-6 was probably associated with non-cardioembolic AIS. To further explore its potential involvement in pathophysiological processes in AIS, we analyzed an AIS dataset from the GEO to extract 782 DEGs that may be AIS-related and TNFAIP6correlated genes. Previous studies have confirmed that TSG-6 was abundantly expressed in human monocyte-derived macrophages (7) and TSG-6 is a secretory protein (6). Here, 
these samples were derived from peripheral blood mononuclear cells (PBMCs), whereas our samples were peripheral plasma. Therefore, we hypothesize that the expression of TSG-6 in PBMCs might roughly match with their plasma levels in the peripheral plasma, or say, between the two, there might exist a similar trend. Subsequently, pathway and function analysis indicated that the DEGs were associated with response to immunoinflammatory response, environmental information processing, atherosclerosis, production and activity of cytokine including chemokine, leukocyte functional activity, and the like. These biological processes above were in accordance with previous research on TSG-6 (6). Further analysis selected 17 targets deemed as closely interacting with TNFAIP6 in AIS, of which CXCL1, IL-8, and EGF were the top 3 with a high degree. CXCL- 1 and IL- 8 are both potent neutrophil chemokines playing noteworthy roles in AIS pathophysiology $(28,29)$. Interestingly, Losy et al. (28) found no increase of serum CXCL1 levels in AIS patients within $24 \mathrm{~h}$ after onset, instead advocated CXCL1 local but not systemic production of inflammatory response to AIS. IL-8 is mainly produced by activated monocytes, macrophages, and endothelial cells and acts as a potential polymorphonuclear leukocyte chemotaxis to induce neutrophils to accumulate at ischemic sites, thereby causing inflammation and edema $(29,30)$. It was known that IL-8 was not merely an independent risk factor affecting AIS prognosis, but its differences could be detected in peripheral blood (31). Several studies have shown that neutrophil recruitment, higher neutrophil-to-lymphocyte ratio, and neutrophil extracellular traps all predict clinical worsening in AIS (32-34). A previous study suggested that TSG-6 inhibited neutrophil migration via direct interaction with IL-8 in vitro (35). Therefore, we speculate that there might be a connection between TSG-6 and IL- 8 in peripheral blood of noncardioembolic AIS patients. A Spearman correlation analysis demonstrated that TSG-6 levels were positively correlated with NIHSS and ASTRAL score at admission, lesion volume, neutrophil count, neutrophil-to-lymphocyte ratio, and IL-8 level. Among the six positive correlation variables, the IL-8 level had the strongest correlation with the TSG-6 level. Besides, by analyzing the GSE22255 dataset, CXCL8 expression was upregulated in the TNFAIP6-high expression group. These all supported our speculation.

By further comparison, we first raised that elevated TSG-6 levels were associated with 3-month poor prognosis of noncardioembolic AIS, which was similar to the observation that with increased TSG-6 levels, myocardial infarction and major adverse cardiovascular events (MACE) were more likely to occur, including cardiovascular death, heart failure, acute myocardial infarction, post-infarction angina, and ischemic stroke in patients with acute coronary syndrome during a period of 4 years after onset (25). By multivariable logistic analysis, TSG6 remained an independent relevant factor for the functional outcome, which suggested that plasma TSG- 6 level might be an emerging predictor of short-term prognosis in noncardioembolic AIS and could help to screen for high-risk patients who need aggressive monitoring and treatment. Nonetheless, these results may contradict the past perceptions of TSG-6, like diverse tissue protective and anti-inflammatory properties. Yet some protective cytokines in AIS such as interleukin-37 (36) and hepatic growth factor (37) exhibited similar results. Inflammatory processes could concomitantly induce both beneficial and detrimental effects after AIS (5). Elevated TSG-6 level hinted that TSG-6 acting as a compensation index to anti-inflammatory action reflected the severity and fighting ability of adverse inflammatory response. By ROC analysis, two cutoff values of plasma TSG-6 concentration for diagnosis and prognosis were raised. Our best predictive value in non-cardioembolic AIS was slightly lower than the previous study of ACS (9.5 ng/ml) (25). In our study, we considered that the TSG- 6 reference value of prognosis assessment was more obvious than that of diagnosis. This can be directly seen from the specificity of cutoff values for diagnosis (specificity, 0.550) and prognosis (specificity, 0.717) assessment, whereas the sensitivities of the two cutoff values were similar. Having a quite strong correlation between the levels of TSG-6 and IL-8 in our study and the known antagonistic relationship between the two (35), we further studied the possible changes in the ratio of the two for prognosis. Interesting, TSG-6-to-IL-8 ratio significantly decreased in the 3 -month unfavorable outcome group of non-cardioembolic AIS patients than that in the favorable outcome group. Nevertheless, either elevated TSG6 level or IL-8 level may predict poor prognosis (29), and a higher TSG-6-to-IL-8 ratio might get a better outcome. Besides, the cutoff value of TSG-6-to-IL-8 ratio for predicting the 3-month prognosis of non-cardioembolic AIS gained a relatively high true-positive rate and low false-positive rate. Hence, there is a relatively bold guess that a non-cardioembolic AIS individual with a lower TSG-6 level and a higher IL-8 level might get a poorer outcome due to lack of compensatory protection, which needs further consideration and discussion. These results implied several possible feedback or response mechanisms between the two. Although no consensus was made about how much a marker must add to clinical models in terms of discrimination and reclassification improvements, this study demonstrated some supporting evidence of TSG-6 in noncardioembolic AIS diagnosis and the value of prognosis assessment and provided a potential target for noncardioembolic AIS treatment.

Our study has several limitations that should be considered. First, the number of subjects was small and our work lacks longterm clinical outcome data. Second, the study population had geographical limitations, so that the findings should be extrapolated to other populations cautiously. Further studies with larger sample sizes from multiple centers might be more persuasive to validate our findings. Third, plasma TSG-6 concentrations were tested only once at admission, so we were unable to study the variation in plasma TSG-6 levels among noncardioembolic AIS patients. Fourth, in samples for screening IL8 as a related factor, the database samples did not highly match with our samples. Although we explained the reason for selecting this method in our previous discussion, the results are more reliable with more consistent sample types and sources. Fifth, due to limitations of environmental, regional, and other factors, 
we were unable to perform face-to-face follow-up. Differences in cognition, description, and cooperation degree of patients and their relatives increased the bias of outcome assessment. Finally, inflammatory response after AIS is a complicated regulatory network, in which each biomarker potentially influences discrimination, reclassification, and accuracy of prediction. The association between TSG- 6 and other inflammatory factors deserves to explore more.

\section{CONCLUSIONS}

Our findings first demonstrated that plasma TSG-6 level was a novel indicator for non-cardioembolic AIS diagnosis and 3month prognosis. The TSG-6 reference value of prognosis assessment was more obvious than that of diagnosis. Plasma TSG-6 and IL-8 levels manifested positive correlations at the early stage of non-cardioembolic AIS. Underlining some potential response mechanisms of TSG-6 and IL-8, elevated TSG-6-to-interleukin-8 ratio might suggest a 3 -month favorable outcome. These results provide vital clues for both basic study and clinical application of non-cardioembolic AIS. Further research into the relationship between TSG-6 and ischemic stroke is necessary and warranted.

\section{DATA AVAILABILITY STATEMENT}

The datasets used during the current study are available from the corresponding author on reasonable request.

\section{ETHICS STATEMENT}

The studies involving human participants were reviewed and approved by the local Ethics Committee of the First Affiliated Hospital of Harbin Medical University. The patients/participants provided their written informed consent to participate in this study.

\section{REFERENCES}

1. GBD 2019 Stroke Collaborators. Global, Regional, and National Burden of Stroke and its Risk Factors, 1990-2019: A Systematic Analysis for the Global Burden of Disease Study 2019. Lancet Neurol (2021) 20:795-820. doi: 10.1016/S1474-4422(21)00252-0

2. Wu S, Wu B, Liu M, Chen Z, Wang W, Anderson CS, et al. Stroke in China: Advances and Challenges in Epidemiology, Prevention, and Management. Lancet Neurol (2019) 18:394-405. doi: 10.1016/S1474-4422(18)30500-3

3. Virani SS, Alonso A, Benjamin EJ, Bittencourt MS, Callaway CW, Carson AP, et al. Heart Disease and Stroke Statistics-2020 Update a Report From the American Heart Association. Circulation (2020) 141:e139-596. doi: 10.1161/ CIR.0000000000000757

4. Chen GY, Nunez G. Sterile Inflammation: Sensing and Reacting to Damage. Nat Rev Immunol (2010) 10:826-37. doi: 10.1038/nri2873

5. Bonaventura A, Liberale L, Vecchié A, Casula M, Carbone F, Dallegri F, et al. Update on Inflammatory Biomarkers and Treatments in Ischemic Stroke. Int J Mol Sci (2016) 17:1967. doi: 10.3390/ijms17121967

\section{AUTHOR CONTRIBUTIONS}

YQ prepared the study protocol; collected, analyzed, and interpreted the data; and prepared the manuscript. FY and FM collected and analyzed the data. XC, QZ, and TY collected the data. SW interpreted the data. YP prepared the study protocol, analyzed and interpreted the data, and supervised the study. All authors read and approved the final manuscript.

\section{FUNDING}

This research was supported by grants from the Opening Foundation of State Key Laboratory of Cognitive Neuroscience and Learning, Beijing Normal University (CNLZD1303 and CNLZD1701) and the National Natural Science Foundation of China (No.32030045).

\section{ACKNOWLEDGMENTS}

We thank Dr. Mingsha Zhang from the State Key Laboratory of Cognitive Neuroscience and Learning, Beijing Normal University for providing helpful comments.

\section{SUPPLEMENTARY MATERIAL}

The Supplementary Material for this article can be found online at: https://www.frontiersin.org/articles/10.3389/fimmu.2022. 713379/full\#supplementary-material

Supplementary Figure 1 | Standard curve of TSG-6 and IL-8.

Supplementary Table 1 | Patient's characteristics stratified by quartiles of TSG-6 plasma Concentration.

Supplementary Data Sheet 1 | Differently expressed genes correlated with TNFAIP6 in AIS.

Supplementary Table 2 | AIS-related targets with closely relevant to TNFAIP6/ TSG-6.

6. Day AJ, Milner CM. TSG-6: A Multifunctional Protein With AntiInflammatory and Tissue-Protective Properties. Matrix Biol (2019) 7879:60-83. doi: 10.1016/j.matbio.2018.01.011

7. Watanabe R, Watanabe H, Takahashi, Kojima M, Konii H, Watanabe K, et al. Atheroprotective Effects of Tumor Necrosis Factor-Stimulated Gene-6. JACC Basic Transl Sci (2016) 1:494-509. doi: 10.1016/j.jacbts.2016.07.008

8. Wang SK, Xie J, Green LA, McCready RA, Motaganahalli RL, Fajardo A, et al. TSG-6 Is Highly Expressed in Human Abdominal Aortic Aneurysms. J Surg Res (2017) 220:311-9. doi: 10.1016/j.jss.2017.06.078

9. Lin Q, Zhao S, Zhou L, Fang X, Fu Y, Huang Z. Mesenchymal Stem Cells Transplantation Suppresses Inflammatory Responses in Global Cerebral Ischemia: Contribution of TNF- $\alpha$-Induced Protein 6. Acta Pharmacol Sinica (2013) 34:784-92. doi: 10.1038/aps.2012.199

10. Al'Qteishat A, Gaffney J, Krupinski J, Rubio F, West D, Kumar S, et al. Changes in Hyaluronan Production and Metabolism Following Ischaemic Stroke in Man. Brain (2006) 129:2158-76. doi: 10.1093/brain/awl139

11. Warlow C, Sudlow C, Dennis M, Wardlaw J, Sandercock P. Stroke. Lancet (2003) 362:1211-24. doi: 10.1016/S0140-6736(03)14544-8 
12. Higgins JPT, Thompson SG, Deeks JJ, Altman DG. Measuring Inconsistency in Meta-Analyses. BMJ (2003) 327:557-60. doi: 10.1136/bmj.327.7414.557

13. Brott T, Adams HPJr, Olinger CP, Marler JR, Barsan WG, Biller J, et al. Measurements of Acute Cerebral Infarction: A Clinical Examination Scale. Stroke (1989) 20:864-70. doi: 10.1161/01.str.20.7.864

14. Ntaios G, Faouzi M, Ferrari J, Lang W, Vemmos K, Michel P. An Integer-Based Score to Predict Functional Outcome in Acute Ischemic Stroke: The ASTRAL Score. Neurology (2012) 78:1916-22. doi: 10.1212/WNL.0b013e318259e221

15. Adams HPJr, Bendixen BH, Kappelle LJ, Biller J, Love BB, Gordon DL, et al. Classification of Subtype of Acute Ischemic Stroke. Definitions for Use in a Multicenter Clinical Trial. TOAST. Trial of Org 10172 in Acute Stroke Treatment. Stroke (1993) 24:35-41. doi: 10.1161/01.str.24.1.35

16. Sims JR, Gharai LR, Schaefer PW, Vangel M, Rosenthal ES, Lev MH, et al. ABC/2 for Rapid Clinical Estimate of Infarct, Perfusion, and Mismatch Volumes. Neurology (2009) 72:2104-10. doi: 10.1212/WNL.0b013e3181aa5329

17. Zaidat OO, Yoo AJ, Khatri P, Tomsick TA, von Kummer R, Saver JL, et al. Cerebral Angiographic Revascularization Grading (CARG) Collaborators, STIR Revascularization Working Group and STIR Thrombolysis in Cerebral Infarction (TICI) Task Force. Recommendations on Angiographic Revascularization Grading Standards for Acute Ischemic Stroke: A Consensus Statement. Stroke (2013) 44:2650-63. doi: 10.1161/STROKEAHA.113.001972

18. Barrett T, Wilhite SE, Ledoux P, Evangelista C, Kim IF, Tomashevsky M, et al. NCBI GEO: Archive for Functional Genomics Data Sets-Update. Nucleic Acids Res (2013) 41:D991-5. doi: 10.1093/nar/gks1193

19. Ritchie ME, Phipson B, Wu D, Hu Y, Law CW, Shi W, et al. Limma Powers Differential Expression Analyses for RNA-Sequencing and Microarray Studies. Nucleic Acids Res (2015) 43:e47. doi: 10.1093/nar/gkv007

20. Zhou Y, Zhou B, Pache L, Chang M, Khodabakhshi AH, Tanaseichuk O, et al. Metascape Provides a Biologist-Oriented Resource for the Analysis of SystemsLevel Datasets. Nat Commun (2019) 10:1523. doi: 10.1038/s41467-019-09234-6

21. Szklarczyk D, Morris JH, Cook H, Kuhn M, Wyder S, Simonovic M, et al. The STRING Database in 2017: Quality-Controlled Protein-Protein Association Networks, Made Broadly Accessible. Nucleic Acids Res (2017) 45:D362-8. doi: 10.1093/nar/gkw937

22. Shannon P, Markiel A, Ozier O, Baliga NS, Wang JT, Ramage D, et al. Cytoscape: A Software Environment for Integrated Models of Biomolecular Interaction Networks. Genome Res (2003) 13:2498-504. doi: 10.1101/gr.1239303

23. Bonita R, Beaglehole R. Recovery of Motor Function After Stroke. Stroke (1988) 19:1497-500. doi: 10.1161/01.str.19.12.1497

24. Libby P, Ridker PM, Maseri A. Inflammation and Atherosclerosis. Circulation (2002) 105:1135-43. doi: 10.1161/hc0902.104353

25. Watanabe R, Sato Y, Ozawa N, Takahashi Y, Koba S, Watanabe T. Emerging Roles of Tumor Necrosis Factor-Stimulated Gene-6 in the Pathophysiology and Treatment of Atherosclerosis. Int J Mol Sci (2018) 19:465. doi: 10.3390/ ijms19020465

26. Ye L, Mora R, Akhayani N, Haudenschild CC, Liau G. Growth Factor and Cytokine-Regulated Hyaluronan-Binding Protein TSG-6 Is Localized to the Injury-Induced Rat Neointima and Confers Enhanced Growth in Vascular Smooth Muscle Cells. Circ Res (1997) 81:289-96. doi: 10.1161/01.res.81.3.289

27. Wang S, Hu S, Zhang Q, Xia A, Jiang Z, Chen X. Mesenchymal Stem Cells Stabilize Atherosclerotic Vulnerable Plaque by Anti-Inflammatory Properties. PloS One (2015) 10(8):e0136026. doi: 10.1371/journal.pone.0136026

28. Losy J, Zaremba J, Skrobański P. CXCL1 (GRO-Alpha) Chemokine in Acute Ischaemic Stroke Patients. Folia Neuropathol (2005) 43:97-102.
29. Kozak HH, Uğuz F, Kılınç İ, Uca AU, Tokgöz OS, Akpınar Z, et al. Delirium in Patients With Acute Ischemic Stroke Admitted to the Non-Intensive Stroke Unit: Incidence and Association Between Clinical Features and Inflammatory Markers. Neurol Neurochir Pol (2017) 51:38-44. doi: 10.1016/j.pjnns. 2016.10.004

30. Huttunen K, Siponen T, Salonen I, Yli-Tuomi T, Aurela M, Dufva H, et al. Low-Level Exposure to Ambient Particulate Matter Is Associated With Systemic Inflammation in Ischemic Heart Disease Patients. Environ Res (2012) 116:44-51. doi: 10.1016/j.envres.2012.04.004

31. Zhang L, Xu D, Zhang T, Hou W, Yixi L. Correlation Between Interleukin-6, Interleukin-8, and Modified Early Warning Score of Patients With Acute Ischemic Stroke and Their Condition and Prognosis. Ann Palliat Med (2021) 10:148-55. doi: 10.21037/apm-20-2200

32. Perez-de-Puig I, Miró-Mur F, Ferrer-Ferrer M, Gelpi E, Pedragosa J, Justicia C, et al. Neutrophil Recruitment to the Brain in Mouse and Human Ischemic Stroke. Acta Neuropathol (2015) 129:239-57. doi: 10.1007/s00401-014-1381-0

33. Ferro D, Matias M, Neto J, Dias R, Moreira G, Petersen N, et al. NeutrophilTo-Lymphocyte Ratio Predicts Cerebral Edema and Clinical Worsening Early After Reperfusion Therapy in Stroke. Stroke (2021) 52:859-67. doi: 10.1161/ STROKEAHA.120.032130

34. Vallés J, Lago A, Santos MT, Latorre AM, Tembl JI, Salom JB, et al. Neutrophil Extracellular Traps Are Increased in Patients With Acute Ischemic Stroke: Prognostic Significance. Thromb Haemost (2017) 117:1919-29. doi: 10.1160/ TH17-02-0130

35. Dyer DP, Thomson JM, Hermant A, Jowitt TA, Handel TM, Proudfoot AEI, et al. TSG-6 Inhibits Neutrophil Migration via Direct Interaction With the Chemokine CXCL8. J Immunol (2014) 192:2177-85. doi: 10.4049/ jimmunol.1300194

36. Zhang F, Zhu T, Li H, He Y, Zhang Y, Huang N, et al. Plasma Interleukin-37 Is Elevated in Acute Ischemic Stroke Patients and Probably Associated With 3Month Functional Prognosis. Clin Interv Aging (2020) 15:1285-94. doi: 10.2147/CIA.S230186

37. Zhu Z, Xu T, Guo D, Huangfu X, Zhong C, Yang J, et al. Serum Hepatocyte Growth Factor Is Probably Associated With 3-Month Prognosis of Acute Ischemic Stroke. Stroke (2018) 49:377-83. doi: 10.1161/STROKEAHA. 117.019476

Conflict of Interest: The authors declare that the research was conducted in the absence of any commercial or financial relationships that could be construed as a potential conflict of interest.

Publisher's Note: All claims expressed in this article are solely those of the authors and do not necessarily represent those of their affiliated organizations, or those of the publisher, the editors and the reviewers. Any product that may be evaluated in this article, or claim that may be made by its manufacturer, is not guaranteed or endorsed by the publisher.

Copyright (c) 2022 Qu, Yang, Meng, Chen, Zhang, Yu, Wen and Pan. This is an open-access article distributed under the terms of the Creative Commons Attribution License (CC BY). The use, distribution or reproduction in other forums is permitted, provided the original author(s) and the copyright owner(s) are credited and that the original publication in this journal is cited, in accordance with accepted academic practice. No use, distribution or reproduction is permitted which does not comply with these terms. 